Bartling, Nachtrag zu der Abhandlung: Condensation ete. 37

\begin{tabular}{lccccc} 
& Berechnet fuir & \multicolumn{5}{c}{ Gefunden } \\
\cline { 3 - 7 } & $\mathrm{C}_{4} \mathrm{H}_{6} \mathrm{~N}_{4} \mathrm{O}_{2}$ & I. & II. & III. & IV. \\
$\mathrm{C}$ & 33,80 & 33,47 & 33,33 & - & - \\
$\mathrm{H}$ & 4,23 & 4,44 & 4,32 & - & - \\
$\mathrm{N}$ & 39,43 & - & - & 38,77 & $\mathbf{3 8 , 7 7}$
\end{tabular}

Da die von den beiden bekannten Krystallarten des Glycolurils, kleinen Octaëdern und spiessigen Nadeln, ganz abweichende Form auch beim Umkrystallisiren aus heissem Wasser erhalten blieb, so wurde zunächst das Vorliegen eines Isomeren angenommen. Diese Vermuthung musste jedoch aufgegeben werden, als eine vergleichende Löslichkeitsbestimmung genaue Uebereinstimmung ergab. Da nunmehr drei verschiedene Formen des Glycolurils von gleicher Löslichkeit bekannt sind, muss man annehmen, dass sehr geringe Mengen von Verunreinigungen die Wachsthumsgeschwindigkeit der Glycolurilkrystalle nach verschiedenen Richtungen sehr stark zu beeinflussen vermögen.

\title{
II. Nachtrag zu der Abhandlung: Ueber die
} Condensation von Isodialursaure mit Thioharnstoff; von Richard Bartling.

(Mitgetheilt von Robert Behrend.)

Durch Oxydation eines aus Isodialursäure und Thiobarnstoff gewonnenen Condensationsproductes $\mathrm{C}_{16} \mathrm{H}_{26} \mathrm{~N}_{12} \mathrm{O}_{11} \mathrm{~S}_{3}$ mit Salpetersäure hatte C. v. Vogel ${ }^{1}$ ) eine Verbindung $\mathrm{C}_{4} \mathrm{H}_{5} \mathrm{~N}_{3} \mathrm{O}_{5}$ erhalten, deren Identität mit Dilitursäure, $\mathrm{C}_{4} \mathrm{H}_{3} \mathrm{~N}_{3} \mathrm{O}_{5}$, bereits früher vermuthet wurde.

Eine erneute Untersuchung hat diese Vermuthung bestätigt; nur wurde bei der Darstellung des Körpers meist nicht freie Dilitursäure, sondern Ammonsalz gewonnen und nur einmal ein Präparat, dessen Zusammensetzung zwischen derjenigen der freien Säure und der des Ammonsalzes lag. Da die Oxydation des Condensationsproductes mit Salpetersäure

1) Diese Annalen 315, 259. 
sebr stürmisch verläuft and ein beträchtlicher Theil der Substanz völlig zerstört wird, so bietet das Auftreten von Ammoniak nichts Wunderbares und da das dilitursaure Ammon durch Salpetersäure nicht zerlegt wird, muss es natürlich als solches ausfallen. Andererseits kann es nicht auffallen, dass zuweilen als Hauptproduct freie Dilitursäure entsteht, wenn entweder die Zersetzung nicht bis zur Bildung von Ammoniak geht oder das entstandene Ammoniak durch die salpetrige Säure zerstört wird.

Bei einem nach x. Vogel's Angaben angestellten Versuche wurde zunächst ein Präparat erhalten, dessen Analyse folgende Zahlen gab:

I. $0,2251 \mathrm{~g}$ gaben $0,1865 \mathrm{CO}_{2}$ und $0,0914 \mathrm{H}_{2} \mathrm{O}$.

II. $0,1993 \mathrm{~g} \quad, \quad 0,1619 \quad \mathrm{CO}_{\mathrm{g}} \quad, \quad 0,0801 \mathrm{H}_{2} \mathrm{O}$.

III. $0,2299 \mathrm{~g} \quad, \quad 46,3 \mathrm{ccm}$ feuchtes Stickgas bei $21^{\circ}$ und $752,5 \mathrm{~mm}$ Druck.

IV. $0,2016 \mathrm{~g}$ gaben $42,0 \mathrm{ccm}$ feuchtes stickgas bei $23^{\circ}$ und $755 \mathrm{~mm}$ Druck.

\begin{tabular}{|c|c|c|c|c|c|}
\hline \multicolumn{2}{|c|}{ Berechnet für } & \multicolumn{4}{|c|}{ Gefumden } \\
\hline Dilitursäure & diliturs. Ammon & I. & II. & III. & IV. \\
\hline $\mathrm{C}_{4} \mathrm{H}_{3} \mathrm{~N}_{5} \mathrm{O}_{\overline{5}}+3 \mathrm{H}_{2} \mathrm{O}$ & $\mathrm{C}_{4} \mathrm{H}_{6} \mathrm{~N}_{4} \mathrm{O}_{3}$ & & & & \\
\hline 21,15 & 25,26 & 22,59 & 22,15 & - & -- \\
\hline 3,91 & 3,18 & 4,53 & 4,50 & - & - \\
\hline 18,50 & 29,47 & - & - & 22,73 & $23,2 \tilde{0}$ \\
\hline
\end{tabular}

Mit Kalilauge entwickelte das Präparat in der Kälte Ammoniak, nach mehrmaligem Umkrystallisiren aus heissem Wasser besass es die Zusammensetzung des dilitursauren Ammons.

I. $0,1570 \mathrm{~g}$ gaben $0,1444 \mathrm{CO}_{2}$ und $0,0515 \mathrm{H}_{2} \mathrm{O}$.

II. $0,1421 \mathrm{~g} \quad " \quad 0,1329 \quad \mathrm{CO}_{z} \quad " \quad 0,0531 \mathrm{H}_{2} \mathrm{O}$.

III. $0,0941 \mathrm{~g}, 23 \mathrm{ccm}$ feuchtes Stickgas bei $14^{\circ}$ und $764 \mathrm{~mm}$ Druck.

Fin neu dargestelltes Präparat gab, einmal nukrystallisirt, folgende Zahien:

IV. $0,1430 \mathrm{~g}$ gaben $0,1319 \mathrm{~g} \mathrm{CO}_{2}$ und $0,0519 \mathrm{H}_{2} \mathrm{O}$.

V. $0,1198 \mathrm{~g}, 29,5$ cсur feuchtes stickgas bei $9^{0}$ und $760 \mathrm{~mm}$ Druck.

Berechnet für

Gefunden

$\begin{array}{lccccccc} & \mathrm{C}_{4} \mathrm{H}_{6} \mathrm{~N}_{4} \mathrm{O}_{5} & \text { I. } & \text { II. } & \text { III. } & \text { IV. } & \text { V. } & \text { v. Vogel im Mittel } \\ \mathrm{O} & 25,26 & 25,08 & 25,51 & & 25,17 & - & 25,55 \\ \mathrm{H} & 3,18 & 3,63 & 4,15 & - & 4,06 & - & 4,13 \\ \mathrm{~N} & 29,47 & - & - & 28,91 & - & 29,54 & 28,12\end{array}$


Bei allen Analysen wurde der Wasserstoffgehalt, oft auch der Kohlenstoffgehalt $\mathrm{zu}$ hoch, der Stickstoffgehalt meist $\mathrm{zu}$ niedrig gefunden. Es rührt dies daher, dass es auch bei der grössten Vorsicht kaum gelingt zu verhindern, dass Stickstoffoxyde unreducirt entweichen. In Anbetracht dieser Fehlerquelle stimmen auch die von v. Vogel mitgetheilten Analysen des Ammonsalzes seiner Säure hinlänglich auf die Formel des dilitursauren Ammons.

Zur sicheren Identificirung wurde nach den Angaben v. Baeyer's ${ }^{2}$ ) Dilitursäure bereitet und in das Ammonsalz übergeführt. Die krystallographische Untersuchung ergab keinen Unterschied der beiden Präparate. Beide stellten unter dem Mikroskope Prismen dar, welche unter einem Winkel von $138^{\circ}$ (v. Vogel) and $133,4^{0}$ (v. Baeyer) abgeschnitten waren. Die Sehiefe der Auslöschung gegen die Prismenkante wurde zu $32^{\circ}$ (v. Vogel) and $29^{\circ}$ (v. Baeyer) bestimmt.

Die Löslichkeit des Wasser von $25,6^{\circ}$ erwies sich ebenfalls als gleich.

Von dem Präparate von Vogel enthielten:

Nach 14 stündigem Schïtteln $8,962 \mathrm{~g}$ Lösung $0,0083 \mathrm{~g} ; 100 \mathrm{~g}$ Lösung $0,093 \mathrm{~g}$.

Nach 22 stündigem Schütteln 9,99 g Lösung $0,0087 \mathrm{~g} ; 100 \mathrm{~g}$ Lö̈sung $0,087 \mathrm{~g}$.

Von dem Präparate v. Baeyer enthielten:

Nach 14 stïndigem Schütteln $9,82 \mathrm{~g}$ Lösung $0,0088 \mathrm{~g} ; 100 \mathrm{~g}$ Lösung $0,0090 \mathrm{~g}$.

Nach 22 stündigem Schütteln 9,91 g Lösung 0,0093 g; $100 \mathrm{~g}$ Lösung $0,0094 \mathrm{~g}$.

Mit Ferrosulfatlösung gaben beide Präparate das von v. Baeyer ${ }^{3}$ ) beschriebene Ferrosalz in Gestalt feiner, sehr schwer löslicher Nädelchen.

Weiter wurde das Barytsalz durch Fällung mit Baryumacetat dargestellt. Aus beiden Präparaten wurde es in feinen

) Diese Annalen 130, 140 .

3) Diese Annalen 127, 218. 
Nadeln gewonnen, welche in heissem Wasser gelöst, auf Zusatz von Chlorbaryumlösung das charakteristische Doppelsalz ${ }^{4}$ ) mit Chlorbaryum lieferten.

Die Krystalle des letzteren zeigten sehr regelmässige Prismenform, vereinzelt fanden sich gypsähnliche Zwillinge. Die Auslöschung lag bei beiden Präparaten parallel und scnkrecht zur Prismenkante; im convergenten polarisirten Lichte zeigten beide ein Curvensystem ähnlich dem, wie es zweiaxige Krystalle senkrecht zur optischen Axe zeigen.

Das nach v. Vogel's Angaben gewonnene Präparat wurde analysirt.

0,1728 g gaben $0,1110 \mathrm{BaSO}_{4}$.

$0,2142 \mathrm{~g}, \quad 0,0856 \mathrm{AgCl}$.

Berechnet für Gefunden

\begin{tabular}{|c|c|}
\hline $\mathrm{C}_{4} \mathrm{H}_{2} \mathrm{~N}_{3} \mathrm{O}_{5} \mathrm{BaC}$ & \\
\hline 37,8 & 37,78 \\
\hline 9,8 & 9,89 \\
\hline
\end{tabular}

Hiernach ist nicht daran zu zweifeln, dass v. Vogel thatsächlich Dilitursäure in Händen gehabt hat.

Die von ihm mitgetheilten Analysen des bei $150^{\circ}$ getrockneten Silbersalzes stimmen ebenso gut auf die Formel des dilitursauren Silbers wie auf die um zwei At. Wasserstoff reichere Formel, wenn man von dem um 0,6 pC, zu hoch gefundenen Wasserstoffgehalt absieht. Die Abweichung ist aus den schon erwähnten Gründen leicht erklärlich.

Das Gleiche gilt von der Analyse der freien Säure, zu welcher v. Vogel jedenfalls, ohne dies besonders anzugeben, bei höherer Temperatur getrocknete Substanz verwendet hat.

\begin{tabular}{|c|c|c|c|c|}
\hline \multirow[b]{2}{*}{$\mathrm{C}$} & \multicolumn{2}{|c|}{ Berechnet für } & \multicolumn{2}{|c|}{ Gefunden } \\
\hline & $\begin{array}{c}\mathrm{C}_{4} \mathrm{H}_{5} \mathrm{~N}_{3} \mathrm{O}_{5} \\
27,43\end{array}$ & $\begin{array}{c}\mathrm{C}_{4} \mathrm{H}_{3} \mathrm{~N}_{3} \mathrm{O}_{5} \\
27,74\end{array}$ & 27,27 & - \\
\hline $\mathrm{H}$ & 2,86 & 1,75 & 2,68 & - \\
\hline $\mathrm{N}$ & 24,00 & 24,28 & 23,27 & 23,70 \\
\hline
\end{tabular}

